Research Article

\title{
SSH: A Tool for Predicting Hydrophobic Interaction of Monoclonal Antibodies Using Sequences
}

\author{
Anthony Mackitz Dzisoo, ${ }^{1}$ Juanjuan Kang, ${ }^{1}$ Pengcheng Yao, ${ }^{1}$ Benjamin Klugah-Brown, ${ }^{2}$ \\ Birga Anteneh Mengesha, ${ }^{1}$ and Jian Huang ${ }^{1}{ }^{1}$ \\ ${ }^{1}$ Center for Informational Biology, University of Electronic Science and Technology of China, Chengdu 611731, China \\ ${ }^{2}$ Brain Connectivity Lab, University of Electronic Science and Technology of China, 611731, China \\ Correspondence should be addressed to Jian Huang; hj@uestc.edu.cn
}

Received 29 February 2020; Revised 28 April 2020; Accepted 13 May 2020; Published 2 June 2020

Guest Editor: Yungang Xu

Copyright (c) 2020 Anthony Mackitz Dzisoo et al. This is an open access article distributed under the Creative Commons Attribution License, which permits unrestricted use, distribution, and reproduction in any medium, provided the original work is properly cited.

\begin{abstract}
Therapeutic antibodies are one of the most important parts of the pharmaceutical industry. They are widely used in treating various diseases such as autoimmune diseases, cancer, inflammation, and infectious diseases. Their development process however is often brought to a standstill or takes a longer time and is then more expensive due to their hydrophobicity problems. Hydrophobic interactions can cause problems on half-life, drug administration, and immunogenicity at all stages of antibody drug development. Some of the most widely accepted and used technologies for determining the hydrophobic interactions of antibodies include standup monolayer adsorption chromatography (SMAC), salt-gradient affinity-capture self-interaction nanoparticle spectroscopy (SGAC-SINS), and hydrophobic interaction chromatography (HIC). However, to measure SMAC, SGAC-SINS, and HIC for hundreds of antibody drug candidates is time-consuming and costly. To save time and money, a predictor called SSH is developed. Based on the antibody's sequence only, it can predict the hydrophobic interactions of monoclonal antibodies (mAbs). Using the leave-one-out crossvalidation, SSH achieved $91.226 \%$ accuracy, $96.396 \%$ sensitivity or recall, $84.196 \%$ specificity, $87.754 \%$ precision, 0.828 Mathew correlation coefficient (MCC), $0.919 f$-score, and 0.961 area under the receiver operating characteristic (ROC) curve (AUC).
\end{abstract}

\section{Introduction}

One of the developing areas in the pharmaceutical industry is therapeutic antibody. The antibody drugs have been used in the treatment of autoimmune diseases, cancer, inflammation, and infectious diseases. However, developing antibody candidates as therapeutic drugs is an expensive and perilous process. Many monoclonal antibody (mAb) candidates failed due to various problems such as poor manufacturability, low stability and solubility, high viscosity, hydrophobicity, and aggregation propensity $[1,2]$.

Though problems mentioned above are due to various reasons, hydrophobic interactions between antibodies themselves or materials of containers have been shown to be the most predominant one. Currently, the available wet lab methods for measuring the hydrophobic interaction of monoclonal antibodies include standup monolayer adsorp- tion chromatography (SMAC), hydrophobic interaction chromatography (HIC), and affinity-capture self-interaction nanoparticle spectroscopy (AC-SINS). SMAC is used to assess colloidal stability of antibodies under different buffer conditions. Antibodies with colloidal instability may be more likely to have nonspecific interactions, and hydrophobic interactions have been suggested to be the main mode of problematic interactions [3]. HIC is used to evaluate the solubility, viscosity, and serum clearance of antibodies, which are mainly influenced by the hydrophobicity of mAbs [4]. AC-SINS is widely used to detect antibody self-association $[5,6]$. Although many physicochemical factors are involved in protein self-association, the presence of hydrophobic moieties on the protein surface is often the primary driver [7]. The methods above have offered a high-throughput solution to developability screening at early-stage antibody drug discovery. However, experimentally screening a large number 
of candidates is still expensive and time-consuming. Computational screening tools are urgently needed.

Computational methods, especially data mining and machine learning techniques, have been widely used in various aspects of biomedical studies [8-17]. The field of antibody drug development is no exception. There are attempts to predict viscosity, developability, crossinteraction, or selfinteraction of antibodies [18-20].

Currently, there are also quite a few existing methods for predicting the hydrophobicity of proteins including mAbs [21-23]. These methods are mostly based on threedimensional structures of proteins. A recent paper by Jain et al. describes a model for predicting delayed retention of antibodies in HIC from the sequence using machine learning [24]. However, no web service is available for this model, just as other published methods for antibody hydrophobicity prediction.

According to our previous working experience on predicting crossinteraction or self-interaction of antibodies, combining data from different but relevant experimental assays gives better results than just relying on a single experimental assay data. In this study, we combine data from SMAC, SGAC-SINS, and HIC that are closely related to the hydrophobicity of antibodies, build a model using machine learning, and construct a web server called SSH. It can predict hydrophobic interactions of antibodies based on just their sequences. The server is freely available at http://i.uestc.edu .cn/eli/cgi-bin/ssh.pl. We believe it can benefit antibody drug screening community by saving time, money, and resources.

\section{Results}

The area under the receiver operating characteristic (ROC) curve (AUC), which is a graphical representation of varying threshold values, explains how well a binary classifier can predict the new data. AUC measures the sensitivity and specificity of the binary classification algorithm, which measures the overall performance of the model; it is referred to as how well a model can predict its negative and positive data. Most binary classification uses AUC as a determinant to show how skewed the classification is toward specificity and sensitivity [25]. The analysis of the ROC curve helps to illustrate how well an individual dataset performs independent of the threshold of prediction [26, 27]. As shown in Figure 1 and Table 1, our models achieved AUC of 0.952, 0.967, 0.965, and 0.961 for SSH1, SSH2, SSH3, and SSH, respectively. AUC represented in the ROC curve further indicates good performance of the classifiers.

Also, the sensitivity or true positive rates (TPR) and specificity or false negative rates (FNR) give the discrepancies in the model; it also shows which data and how many positive and negative data are predicted correctly in the leave-oneout crossvalidation. Our ensemble model SSH predicted correctly $96.396 \%$ and $84.073 \%$ of the positive and negative data, respectively, as shown in Table 1 below.

As shown in Figure 2, the heat map from $f$-scores of 8000 tripeptides of the 3 models, SSH1, SSH2, and SSH3, shows which tripeptide or amino acid contributes more to predictive results.

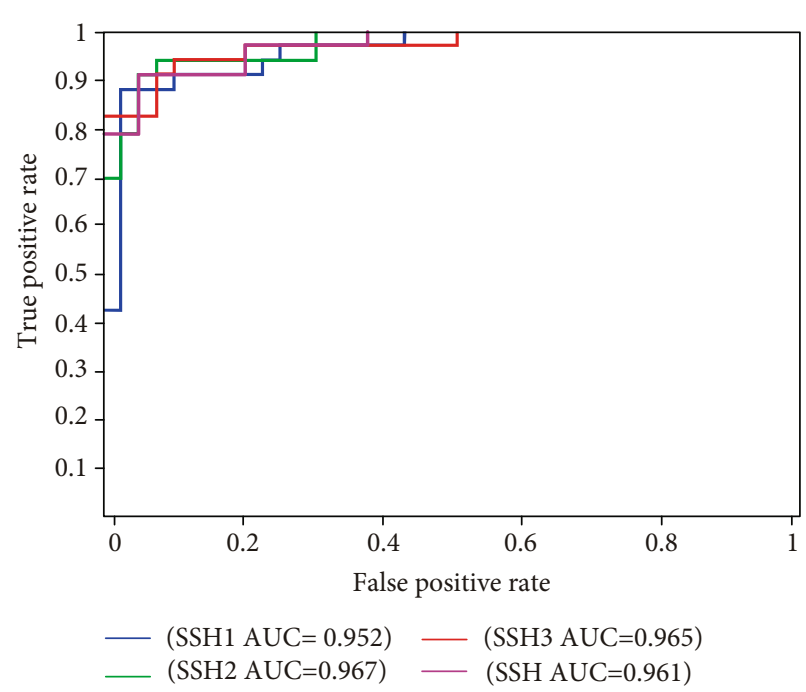

FIgURE 1: ROC and AUC of our model from the leave-one-out crossvalidation.

TABLE 1: Statistical results of the SSH.

\begin{tabular}{lcccc}
\hline & SSH1 & SSH2 & SSH3 & SSH \\
\hline Recall/sensitivity & $97.297 \%$ & $94.595 \%$ & $97.297 \%$ & $96.396 \%$ \\
Specificity & $83.871 \%$ & $87.097 \%$ & $81.300 \%$ & $84.073 \%$ \\
Accuracy & $91.177 \%$ & $92.647 \%$ & $89.855 \%$ & $91.226 \%$ \\
BAC & 0.906 & 0.908 & 0.893 & 0.902 \\
AUC & 0.952 & 0.967 & 0.965 & 0.961 \\
MCC & 0.827 & 0.855 & 0.803 & 0.828 \\
\hline
\end{tabular}

To determine which amino acid gave more predictive values and is the most important to model construction, we calculated the $f$-scores of the tripeptides; the amino acid frequency of the 30 TPC with the best $f$-scores is shown in Figure 3 , which shows tyrosine is the most occurring and important in the model construction. Also, Figure 4 shows 30 tripeptides with the best $f$-scores.

\section{Discussion}

In this study, machine learning methods were employed to predict the hydrophobic interactions of antibodies. Improper hydrophobic interactions can cause a lot of problems in antibody drug development. The datasets were constructed according to three biophysical assay values. Our model SSH was trained with TPC and achieved an accuracy of $91.226 \%$ using the leave-one-out crossvalidation, with $96.396 \%$ sensitivity or recall, $84.100 \%$ specificity, $87.754 \%$ precision, 0.828 MCC, $0.919 f$-score, and 0.961 AUC. This work provides the ability to accurately predict flags in antibodies caused by hydrophobic interactions and will help facilitate the ease of development and subsequent drug manufacturing.

From our analysis, tyrosine, serine, threonine, and glycine are the four amino acids with the best $f$-scores or the best predictive amino acids; tyrosine residues are vastly present in the active sites of antibodies [28, 29]. 

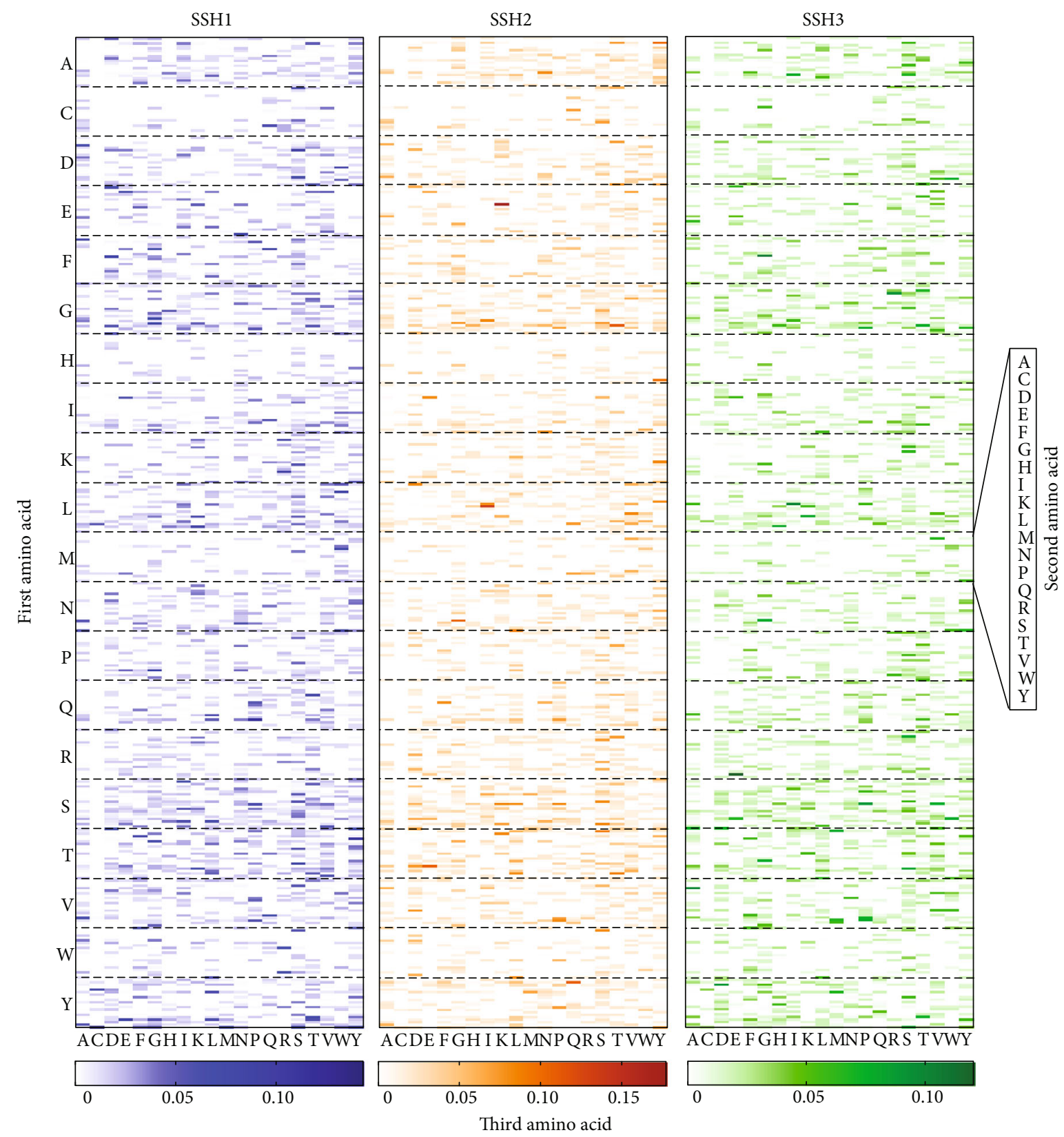

FIgURE 2: Heat map of the 131 observations in the leave-one-out crossvalidation.

The $96.396 \%$ sensitivity proved the ability of our model to correctly identify those antibodies with "flags," and the $84.10 \%$ specificity proved the ability of our model to correctly identify those antibodies without "flags." The AUC of 0.961 and MCC of 0.828 proved that our model is good at predicting both the negative and positive data.

To determine the prediction results or SSH, a voting method is used depending on the $p$ value of the three models SSH1, SSH2, and SSH3. SSH predicts the probability of each antibody input. The higher the probability is, the more likely the antibody is to have hydrophobicity problems. Also, users can set the threshold between 0 and 1 , with a higher threshold meaning stricter validation.

In summary, the predictor enhanced our knowledge of how problems in antibodies could be detected for cost and time reduction; also, the work shows the possibility of virtual screening antibody drug candidates in a large scale at the early stage of development.

\section{Dataset and Methods}

4.1. Dataset. The antibody dataset was downloaded from the supplementary materials of the article published by Jain et al. [30]. The dataset includes 48 approved antibodies and 89 antibodies in the phase 2 and phase 3 clinical trials with 6 entries excluded due to conflicting sequences. The remaining 131 antibodies were used to develop SSH. The $10 \%$ threshold was employed as in Jain et al. to determine if the antibody has 1 or more "flags" (problems) according to the 3 assays, i.e., SMAC, SGAC-SINS, and HIC [30]. An antibody is labeled 


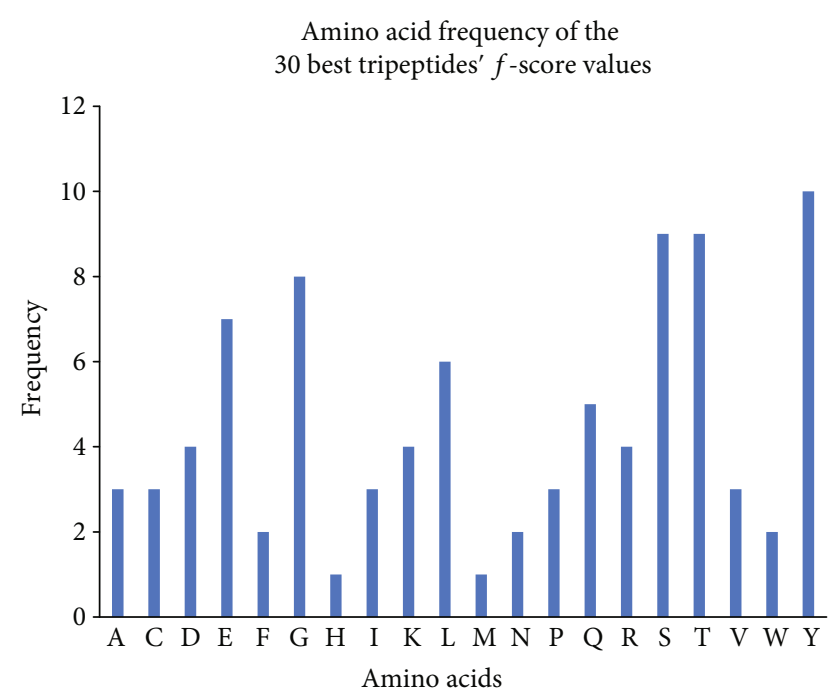

Figure 3: Amino acid frequency from the 30 best tripeptides' $f$ -scores.

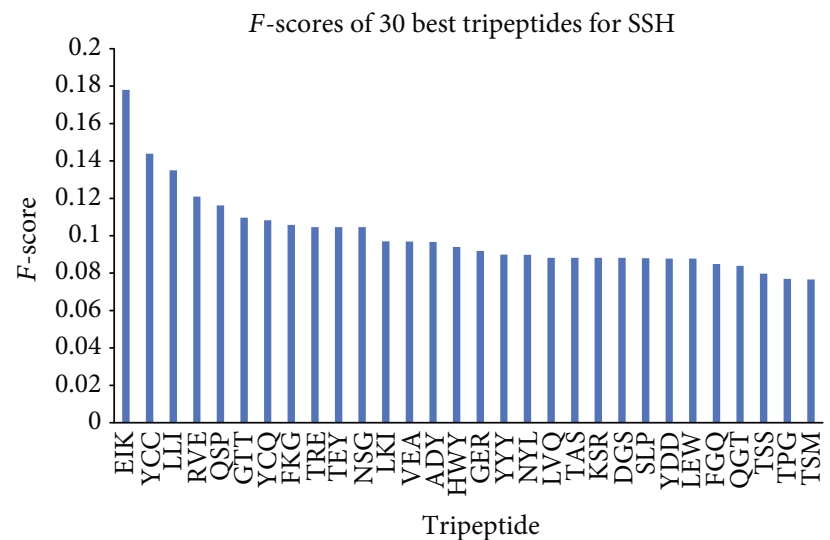

Figure 4: 30 tripeptides with the best $f$-scores.

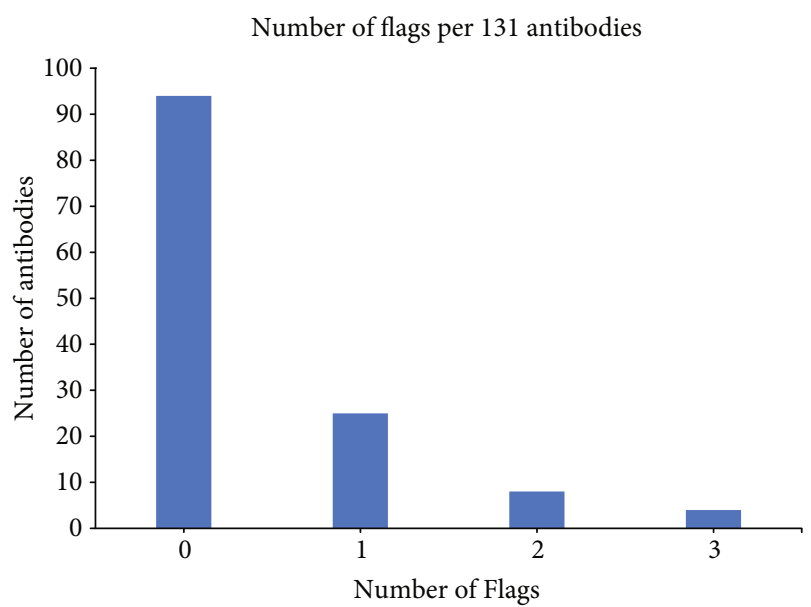

Figure 5: Number of antibodies per flag of 131 antibodies.

with a flag if one of its above assay values falls within the worst $10 \%$ threshold. On the other hand, the antibody with an assay value that falls outside the threshold value is deemed
TABLE 2: Threshold values of 3 assays [30].

\begin{tabular}{lcc}
\hline Assays & $\begin{array}{c}\text { Threshold } \\
\text { values }\end{array}$ & Units (flags) \\
\hline $\begin{array}{l}\text { Standup monolayer adsorption } \\
\text { chromatography (SMAC) }\end{array}$ & 12.8 & $\begin{array}{c}\text { Retention time } \\
(\text { min })(>)\end{array}$ \\
$\begin{array}{l}\text { Salt-gradient affinity-capture } \\
\text { self-interaction nanoparticle } \\
\text { spectroscopy (SGAC-SINS) }\end{array}$ & 370 & $\begin{array}{c}\text { Salt concentration } \\
(\mathrm{mM})(<)\end{array}$ \\
$\begin{array}{l}\text { Hydrophobic interaction } \\
\text { chromatography (HIC) }\end{array}$ & 11.7 & $\begin{array}{c}\text { Retention time } \\
(\min )(>)\end{array}$ \\
\hline
\end{tabular}

without a flag. Of the 131 antibodies, 94 have no flag, 25 have exactly one flag, 8 antibodies have exactly two flags, and 4 antibodies have exactly three flags, as shown in Figure 5. The antibodies with no flags were used as the negative dataset, and those antibodies with at least one flag were used as the positive dataset. The datasets are not balanced, since there are more negative entries. To solve this problem, we split the negative dataset randomly into three subsets with 31,31 , and 32 antibodies, respectively. Each subset is paired with the positive dataset, and 3 models were trained and called SSH1, SSH2, and SSH3. An ensemble method is used to combine the 3 models into SSH using the voting method.

4.2. Features and Feature Selection. The tripeptide composition (TPC) is widely used to convert the sequences to vectors as TPC helps to reflect the sequence order and total amino acid composition. TPC has better predictive results than a single amino acid and a dipeptide composition [19, 31]. The method for extracting TPC is shown as

$$
\operatorname{TPC}(i)=\frac{x(i)}{\sum_{i=1}^{8000} x(i)},
$$

where $i$ equals one of the 8000 tripeptide compositions and $x(i)$ denotes the number of residues of each type of sequence.

From TPC, the best features were selected from the 8000 features using (fselet.py) in LIBSVM, which made use of $f$ -scores to obtain the optimal features; given two sets of real numbers, $f$-score technique measures the discrimination of the two sets [32]. Finally, 313, 315, and 315 features were used to build models SSH1, SSH2, and SSH3, respectively.

4.3. The Threshold Method. The threshold method is used to generate indexes for grouping the negative and positive datasets, as shown in Table 2. The $10 \%$ threshold is calculated as

$$
\text { Thresholdval }=\frac{\sum_{i=N}^{i=0} X_{(N-y+i)}}{y} \text {, }
$$

where $N$ is the number of antibodies, $X(i)$ is the $i$ th antibody's assay value, and $y=10 \%(N)$.

4.4. Support Vector Machine (SVM). The support vector machine (SVM) orders data by finding the best hyperplane separating two classes of data points. The best hyperplane for an SVM means the one with the largest margin between the two classes. The margin means the maximal width of 


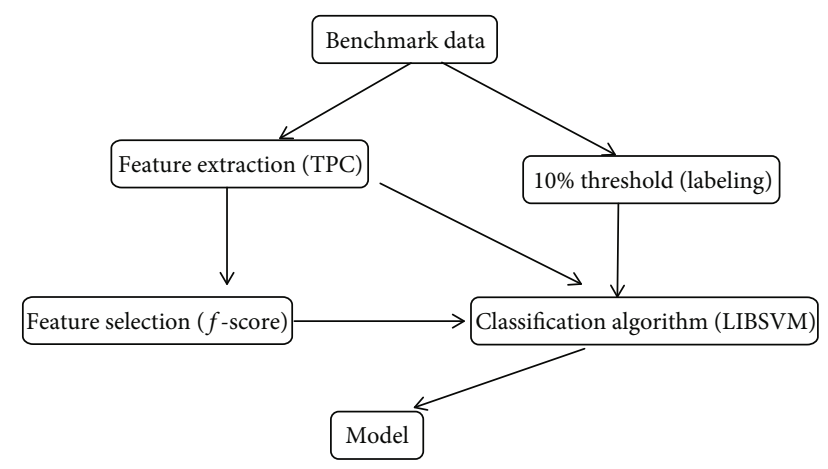

Figure 6: Benchmark of SSH.

the slab parallel to the hyperplane that has no interior data points. SVM is a machine learning method for classifying binary data and multiple class data. It is an effective machine learning method for supervised pattern recognition based on the theory of statistical learning. SVM has been widely used in the field of bioinformatics. We employed LIBSVM [33] with the following parameters: $C=2,128$, and 512 and $g=$ $0.0078125,0.0001220703125$, and 0.0001220703125 for SSH1, SSH2, and SSH3, respectively, for the development of SSH using "RBF" kernel with the leave-one-out crossvalidation [33] .

4.5. Performance Evaluation of SSH. To measure the performance of the SSH, the leave-one-out crossvalidation was used with these measurement parameters, namely, sensitivity (SN), specificity (SP), Mathew correlation coefficient (MCC), accuracy (ACC), and AUC.

Precision is the proportion of the predicted positive cases that were correct. However, accuracy is not only the true measure of a model; the Mathew correlation coefficient (MCC) should be included to evaluate the prediction performance of the developed tool (Equation (6)). MCC is another measure used in machine learning for judging the quality of binary classifications and is considered to be the most robust parameter of any class prediction method.

$$
\begin{aligned}
\mathrm{SN} / \text { Recall } & =\frac{\mathrm{TP}}{\mathrm{TP}+\mathrm{FN}}, \\
\mathrm{SP} & =\frac{\mathrm{TN}}{\mathrm{TN}+\mathrm{FP}}, \\
\mathrm{ACC} & =\frac{\mathrm{TP}+\mathrm{TN}}{\mathrm{TP}+\mathrm{FN}+\mathrm{TN}+\mathrm{FP}}, \\
\mathrm{MCC} & =\frac{\mathrm{TP} * \mathrm{TN}-\mathrm{FP} * \mathrm{FN}}{\sqrt{(\mathrm{TP}+\mathrm{FP})(\mathrm{TP}+\mathrm{FN})(\mathrm{TN}+\mathrm{FP})(\mathrm{TN}+\mathrm{FN})}},
\end{aligned}
$$

where TP is true positive, $\mathrm{FN}$ is false negative, $\mathrm{TN}$ is true negative, and $\mathrm{FP}$ is false positive.

Figure 6 shows the benchmark of the SSH; the 10\% threshold method is used for labeling the negative and positive data.
Abbreviations
AUC: $\quad$ Area under the receiver operating character-
CDR: $\quad$ istic curve
HIC: $\quad$ Hydrophobic interaction chromatography
MCC: $\quad$ Mathew correlation coefficient
ROC: $\quad$ Receiver operating characteristic curve
SGAC-SINS: Salt-gradient affinity-capture self-interaction nanoparticle spectroscopy
SMAC: $\quad$ Standup monolayer adsorption
chromatography
SVM: $\quad$ Support vector machine
TPC: $\quad$ The tripeptide composition
BAC: Balance accuracy.

\section{Data Availability}

The data used to support the findings of this study are available freely at http://i.uestc.edu.cn/eli/sshdownload.html.

\section{Conflicts of Interest}

The authors declare no conflict of interest.

\section{Authors' Contributions}

The contributions of the authors involved in this study are as follows: conceptualization, methodology, writing-original draft preparation, and formal analysis, A.M.D.; validation, A.M.D., J.K., P.Y., and B.K.; writing-review and editing, B.A.M. and B.K.; and supervision, J.H.

\section{Acknowledgments}

This work was supported by the National Natural Science Foundation of China (grant no. 61571095).

\section{References}

[1] O. Leavy, "Therapeutic antibodies: past, present and future," Nature reviews Immunology, vol. 10, no. 5, p. 297, 2010.

[2] J. K. Liu, "The history of monoclonal antibody development Progress, remaining challenges and future innovations," Annals of medicine and surgery, vol. 3, no. 4, pp. 113-116, 2014.

[3] N. Kohli, N. Jain, M. L. Geddie, M. Razlog, and L. Xu, "A novel screening method to assess developability of antibody-like molecules," $m A b s$, vol. 7, no. 4, pp. 752-758, 2015.

[4] P. Estep and I. Caffry, "An alternative assay to hydrophobic interaction chromatography for high-throughput characterization of monoclonal antibodies," $m A b s$, vol. 7 , no. 3, pp. 553-561, 2015.

[5] Y. Liu, I. Caffry, J. Wu et al., "High-throughput screening for developability during early-stage antibody discovery using self-interaction nanoparticle spectroscopy," $m A b s$, vol. 6, no. 2, pp. 483-492, 2014.

[6] S. V. Sule, M. Sukumar, W. F. Weiss, A. M. Marcelino-Cruz, T. Sample, and P. M. Tessier, "High-throughput analysis of concentration-dependent antibody self-association," Biophysical journal, vol. 101, no. 7, pp. 1749-1757, 2011. 
[7] M. Hebditch, A. Roche, R. A. Curtis, and J. Warwicker, "Models for antibody behavior in hydrophobic interaction chromatography and in self-association," Journal of Pharmaceutical Sciences, vol. 108, no. 4, pp. 1434-1441, 2019.

[8] C. Pian, G. Zhang, F. Li, and X. Fan, "MM-6mAPred: identifying DNA N6-methyladenine sites based on Markov model," Bioinformatics, vol. 36, no. 2, pp. 388-392, 2019.

[9] W. Chen, H. Lv, F. Nie, and H. Lin, "i6mA-Pred: identifying DNA N6-methyladenine sites in the rice genome," Bioinformatics, vol. 35, no. 16, pp. 2796-2800, 2019.

[10] C. Q. Feng, Z. Y. Zhang, X. J. Zhu et al., "iTerm-PseKNC: a sequence-based tool for predicting bacterial transcriptional terminators," Bioinformatics, vol. 35, no. 9, pp. 1469-1477, 2019.

[11] B. He, H. Chen, N. Li, and J. Huang, "SAROTUP: a suite of tools for finding potential target-unrelated peptides from phage display data," International Journal of Biological Sciences, vol. 15, no. 7, pp. 1452-1459, 2019.

[12] B. He, J. Kang, B. Ru, H. Ding, P. Zhou, and J. Huang, "SABinder: a web service for predicting streptavidin-binding peptides," BioMed research international, vol. 2016, Article ID 9175143, 8 pages, 2016.

[13] Q. Tang, F. Nie, J. Kang, H. Ding, P. Zhou, and J. Huang, "NIEluter: predicting peptides eluted from HLA class I molecules," Journal of immunological methods., vol. 422, pp. 22-27, 2015.

[14] B. He, G. Chai, Y. Duan et al., "BDB: biopanning data bank," Nucleic acids research, vol. 44, no. D1, pp. D1127-D1132, 2016.

[15] J. Huang, B. Ru, P. Zhu et al., "MimoDB 2.0: a mimotope database and beyond," Nucleic acids research., vol. 40, no. D1, pp. D271-D277, 2011.

[16] N. Li, J. Kang, L. Jiang, B. He, H. Lin, and J. Huang, "PSBinder: a web service for predicting polystyrene surface-binding peptides," BioMed research international, vol. 2017, Article ID 5761517, 5 pages, 2017.

[17] Y. Zhang, T. Liu, L. Chen et al., "RIscoper: a tool for RNARNA interaction extraction from the literature," Bioinformatics, vol. 35, no. 17, pp. 3199-3202, 2019.

[18] N. J. Agrawal and B. Helk, "Computational tool for the early screening of monoclonal antibodies for their viscosities," mAbs., vol. 8, no. 1, pp. 43-48, 2015.

[19] A. M. Dzisoo, B. He, R. Karikari, E. Agoalikum, and J. Huang, "CISI: a tool for predicting cross-interaction or self-interaction of monoclonal antibodies using sequences," Interdisciplinary sciences: computational life sciences, vol. 11, no. 4, pp. 691697, 2019.

[20] T. M. Lauer, N. J. Agrawal, N. Chennamsetty, K. Egodage, B. Helk, and B. L. Trout, "Developability index: a rapid in silico tool for the screening of antibody aggregation propensity," Journal of Pharmaceutical Sciences, vol. 101, no. 1, pp. 102115,2012

[21] M. E. Lienqueo, A. Mahn, G. Navarro et al., "New approaches for predicting protein retention time in hydrophobic interaction chromatography," Journal of molecular recognition : JMR., vol. 19, no. 4, pp. 260-269, 2006.

[22] A. Mahn, M. E. Lienqueo, and J. C. Salgado, "Methods of calculating protein hydrophobicity and their application in developing correlations to predict hydrophobic interaction chromatography retention," Journal of chromatography A, vol. 1216, no. 10, pp. 1838-1844, 2009.
[23] A. T. Hanke, M. E. Klijn, P. D. E. M. Verhaert et al., "Prediction of protein retention times in hydrophobic interaction chromatography by robust statistical characterization of their atomic-level surface properties," Biotechnology progress., vol. 32, no. 2, pp. 372-381, 2016.

[24] T. Jain, T. Boland, A. Lilov et al., "Prediction of delayed retention of antibodies in hydrophobic interaction chromatography from sequence using machine learning," Bioinformatics, vol. 33 , no. 23 , pp. 3758-3766, 2017.

[25] A. P. Bradley, "The use of the area under the ROC curve in the evaluation of machine learning algorithms," Pattern Recognition, vol. 30, no. 7, pp. 1145-1159, 1997.

[26] B. He, H. Chen, and J. Huang, "PhD7Faster 2.0: predicting clones propagating faster from the Ph.D.-7 phage display library by coupling PseAAC and tripeptide composition," PeerJ, vol. 7, p. e7131, 2019.

[27] B. Ru, ‘. T. H. PA, F. Nie, H. Lin, F. B. Guo, and J. Huang, "PhD7Faster: predicting clones propagating faster from the Ph.D.-7 phage display peptide library," Journal of bioinformatics and computational biology, vol. 12, no. 1, article 1450005, 2014.

[28] F. A. Fellouse, P. A. Barthelemy, R. F. Kelley, and S. S. Sidhu, "Tyrosine plays a dominant functional role in the paratope of a synthetic antibody derived from a four amino acid code," Journal of Molecular Biology., vol. 357, no. 1, pp. 100-114, 2006.

[29] F. A. Fellouse, B. Li, D. M. Compaan, A. A. Peden, S. G. Hymowitz, and S. S. Sidhu, "Molecular recognition by a binary code," Journal of Molecular Biology., vol. 348, no. 5, pp. 1153-1162, 2005.

[30] T. Jain, T. Sun, S. Durand et al., "Biophysical properties of the clinical-stage antibody landscape," Proceedings of the National Academy of Sciences of the United States of America, vol. 114, no. 5, pp. 944-949, 2017.

[31] J. Kang, Y. Fang, P. Yao, N. Li, Q. Tang, and J. Huang, "NeuroPP: a tool for the prediction of neuropeptide precursors based on optimal sequence composition," Interdisciplinary sciences, computational life sciences., vol. 11, no. 1, pp. 108-114, 2019.

[32] Y. W. Chen and C. J. Lin, "Combining SVMs with various feature selection strategies," in Feature extraction, vol. 207, pp. 315-324, Springer, Berlin, Heidelberg, 2006.

[33] C.-C. Chang and C.-J. Lin, "LIBSVM," ACM Transactions on Intelligent Systems and Technology, vol. 2, no. 3, pp. 1-27, 2011. 